\title{
Cavitation d'entrefer dans les machines hydrauliques
}

\author{
R. Laborde, P. Chantrel, A. Retailleau \\ a.c.b.-CERG, rue Lavoisier, 38800 Le Pont de Claix
}

\author{
M. Mory \\ Laboratoire des Ecoulements Géophysiques et Industriels/IMG, BP 53X, 38041 Grenoble Cedex
}

\section{Introduction}

Sur une machine hydraulique tournante, de la cavitation apparaît fréquemment dans le jeu entre la tête des aubages et la ceinture fixe. La maîtrise de ce type de cavitation, appelée cavitation d'entrefer, est particulièrement importante, dans la mesure où la cavitation se déclenche souvent sous cette forme dans les machines tournantes, produisant les désagréments généralement associés à ce phénomène : vibrations, bruits, endommagements mécaniques et baisses de performances. Les domaines directement concernés par la cavitation d'entrefer sont les propulseurs marins (propulseurs carénés, pompes hélices, hélices sous tuyère) ainsi qu'un grand nombre de machines hydrauliques industrielles de type pompe ou turbine.

La cavitation d'entrefer est souvent concomitante avec la cavitation de vortex. Toutes deux sont associées à un gradient de pression intrados/extrados. L'écoulement intrados/extrados généré par le gradient de pression traverse l'entrefer et se déverse côté extrados sous la forme d'une nappe cisaillée qui s'enroule en un tourbillon à l'aval dans l'espace interaube.
Les études sur la cavitation de vortex et sur la cavitation d'entrefer, en milieu confiné, sont très nombreuses. Le rapport CERG R. 21.015 (contrat DRET 88/321) en propose une synthèse. Les travaux présentés ici concernent plus particulièrement les effets résultant de modifications de la géométrie de l'entrefer et de la forme des pales. Ils ont été guidés par les études de StrscheletzKy (1955), Novoderezhkin, Karelin \& Demidov (1983) et de Papir \& Golikov (1985), qui ont préconisé, à partir de l'analyse des pertes de charge, un arrondi de l'arête d'entrée de l'entrefer. D'autres modifications possibles de la géométrie de l'entrefer ont été suggérées par GEARHART (1966) (divergence du jeu d'entrefer côté extrados) et par MüLLer (1935) (fixation d'une lame mince en sortie d'entrefer côté extrados). Enfin, un effet de la forme des pales (dévers) sur la cavitation d'entrefer a été relevé par CHANTREL \& LEMONNIER (1984) lors d'essais à NEYRTEC. Les études expérimentales en géométrie et conditions de fonctionnement proches de la réalité industrielle sont en revanche très peu nombreuses. La présente étude est, à notre connaissance, l'une des premières de ce type. Citons également la très récente étude expérimentale de FARrell (1989), davantage orientée sur la cavitation du tourbillon de bout de pale en milieu confiné.

Tip clearance cavitation in hydraulic machines

Experimental results obtained at ACB-CERG concerning tip clearance and vortex cavitation in a pump type rotating machine are presented. The effects of tip clearance geometry, tip clearance gap, blade geometry and pressure gradient between blade and suction faces on these two types of cavitation are considered. The facility also allows to modify the boundary layer structure on the ring belt as well as to control the spectra of cavitation nuclei inside the test section. The analysis relies on visualisations of cavitation patterns and on pressure measurements on the wall of the ring belt. 
Nous présentons ici les résultats d'essais systématiques sur la cavitation d'entrefer, réalisés en 1990 et 1991 à a.c.b.-CERG. Ce travail a été réalisé avec le soutien financier de la DRET (contrats $89 / 403$ et $90 / 518$ ) et en collaboration avec le LEGI/IMG. Les résultats obtenus s'appuient sur deux types de mesures et d'observations :

- des visualisations dans les entrefers et dans les canaux interaube,

- des mesures de pression pariétale sur la ceinture du rotor.

Nous ne donnons ici qu'un compte-rendu partiel des résultats obtenus au cours de l'étude. Les mesures des seuils critiques d'apparition de la cavitation et la comparaison de ces résultats avec les mesures de pression pariétale ne sont pas présentées dans cet article qui tente de mettre en évidence la complémentarité entre les mesures de pression et les visualisations. Cette présentation illustre bien les conclusions principales de l'étude, concernant les modifications géométriques de l'entrefer et des pales.

\section{Paramètres d'influence}

Les paramètres influant sur les cavitations d'entrefer et de vortex, pris en compte dans cette étude, sont essentiellement :

(i) la charge du rotor $\Delta H$ représentative de la différence de pression intrados/extrados: c'est le paramètre générateur par excellence, à partir duquel le nombre de cavitation pertinent est exprimé ;

(ii) la géométrie des entrefers et la hauteur du jeu : quatre géométries d'entrefer ont été étudiées.

a) entrefer à arêtes vives (cas de référence),

b) entrefer arrondi côté intrados,

c) entrefer arrondi côté intrados et divergent côté extrados,

d) entrefer arrondi côté intrados doté d'une lame mince côté extrados.

Dans nos expériences la hauteur du jeu des pales est de 0,5-1 ou $2 \mathrm{~mm}$;

(iii) la géométrie des pales : trois rotors ont été étudiés. Les pales du premier rotor sont «droites». Les pales montées sur les rotors 2 et 3 possèdent un dévers en sens direct ou inverse, respectivement ;

(iv) l'épaisseur et la structure de la couche limite pariétale. Par un dispositif d'aspiration, les caractéristiques de la couche limite sont modifiées dans nos expériences.

\section{Installation expérimentale}

Les essais ont été réalisés dans une veine d'essais concue et implantée pour cette étude sur le tunnel hydrodynamique TH300 de a.c.b.-CERG.

Le débit dans la veine d'essais et la rotation du rotor sont fixés indépendamment. La veine d'essais est pressurisable continûment entre 0 et 3 bars absolus. L'instrumentation du tunnel permet l'injection et le contrôle des

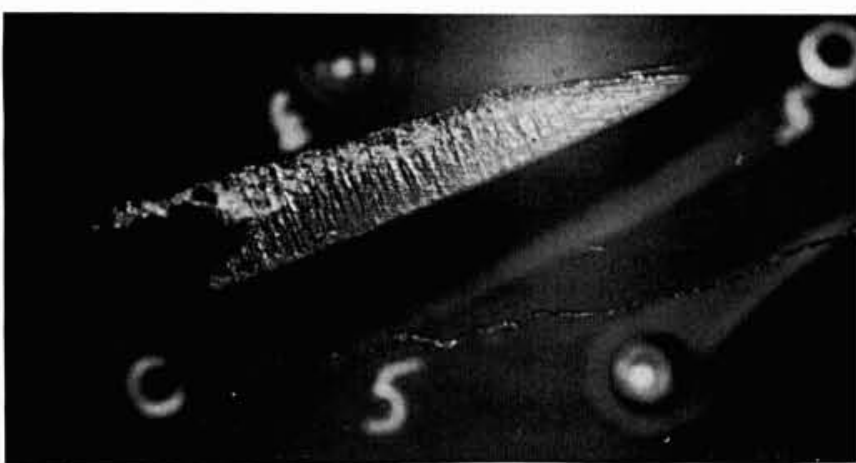

(1a). pale 5: entrefer à arêtes vives

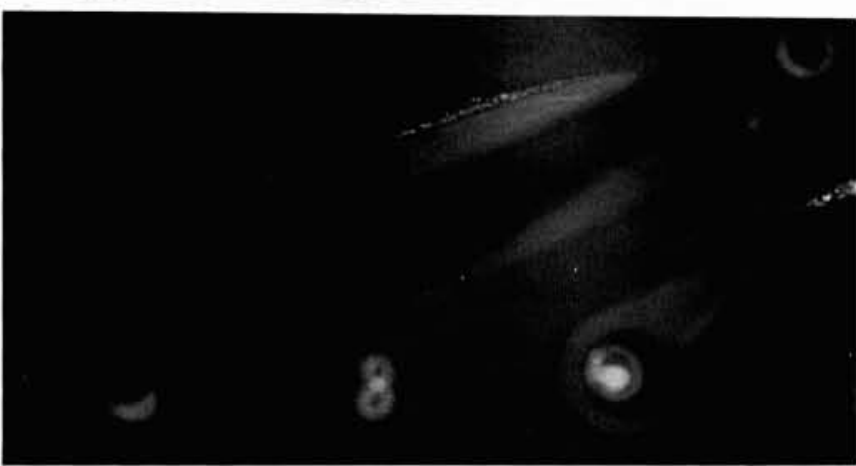

(1b). pale 8: entrefer arrondi côté intrados

1. Visualisations de cavitations d'entrefer et de vortex pour deux géométries d'entrefer (hauteur du jeu : $1 \mathrm{~mm}$ ). Dévers en sens direct (rotor 2 ).

germes. 12 pales sont montées sur le rotor (moyeu: $\phi 290 \mathrm{~mm}$; veine d'essais : $\phi 400 \mathrm{~mm}$ ).

Les principales mesures effectuées pour caractériser le régime de fonctionnement du rotor et du tunnel sont les suivantes :

- débit dans la veine et débit de couche limite aspiré ; - pression statique, charge et vitesse de rotation du rotor ;

- oxygène dissous et spectre de germes ;

— profil de vitesse dans la couche limite.

L'analyse des résultats s'appuie sur des visualisations et des mesures de pression fluctuante sur les parois de la veine d'essai :

- visualisations : la veine d'essai est en plexiglas. Une boîte à eau, située en haut de la veine, permet d'observer sans distorsion optique la cavitation à l'aide d'un éclairage stroboscopique synchronisé ;

— pressions fluctuantes : huit capteurs piézo-résistifs sont disposés sur la paroi de la ceinture en regard des rotors. Les signaux temporels de pression le long des huit cercles parcourus sur la ceinture de la veine par les têtes d'aubages sont numérisés à une fréquence de $31 \mathrm{kHz}$.

Les résultats des essais sont détaillés dans les rapports CERG nं 21.200 et 21.298 


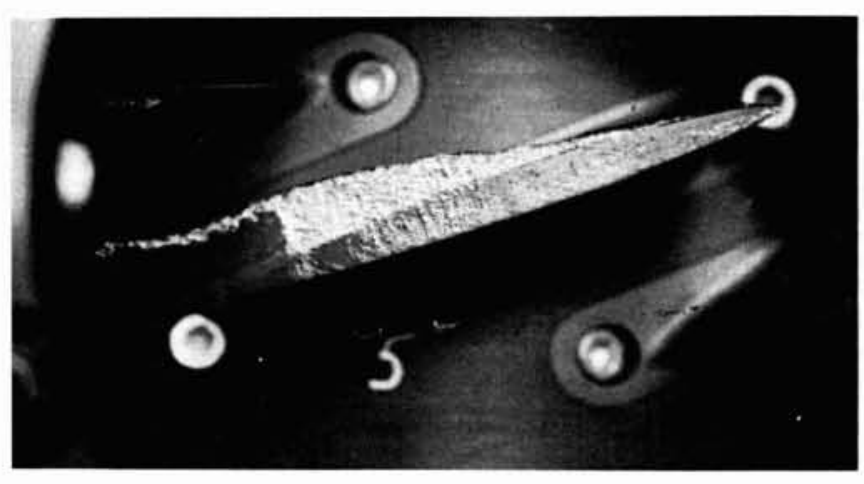

(2a). pale 5: rotor 1, sans dévers.

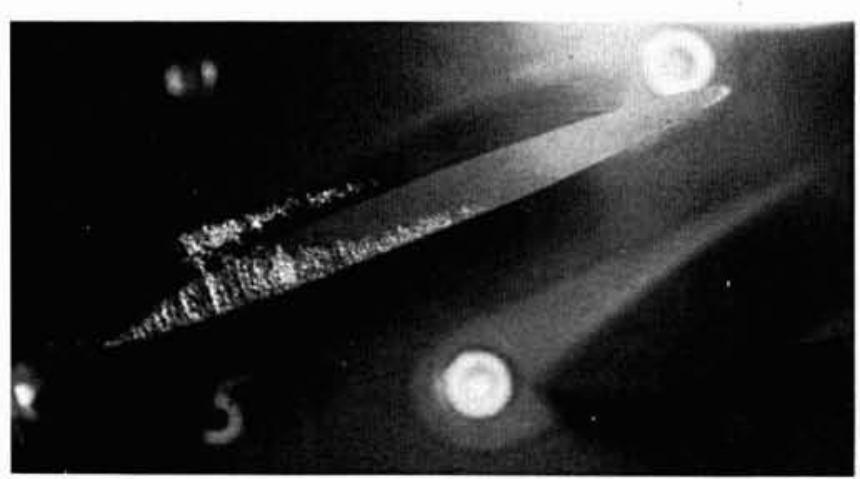

(2b). pale 5: rotor 3, dévers en sens inverse.

2. Effet du dévers sur les cavitations d'entrefer et de vortex pour the pale dentrefer à arêtes vives (hauteur du jeu: $1 \mathrm{~mm}$ ).

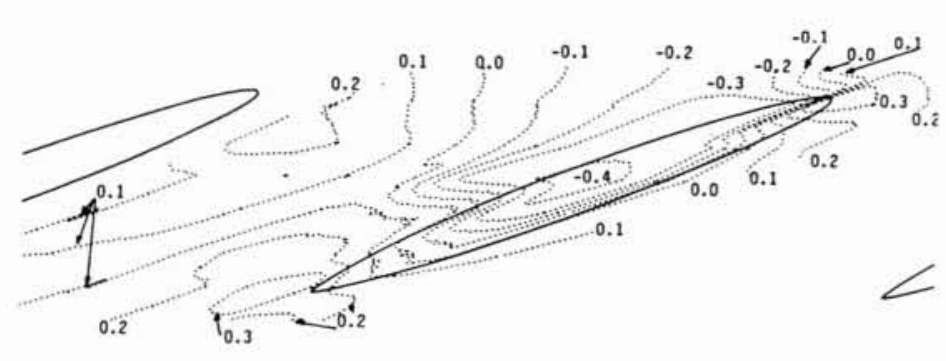

(3a) pale 5. entrefer à arêtes vives

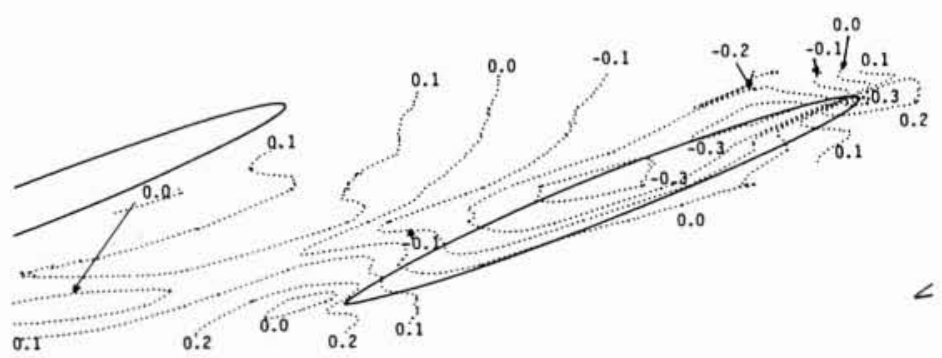

(3b) pale 8: entrefer arrondi côté intrados

3. Champs de pression mesurés sur la ceinture du rotor pour deux géométries d'entrefer (hauteur du jeu: $1 \mathrm{~mm}$ ). Dévers en sens direct (rotor 2).

\section{Résultats expérimentaux}

\section{I. Visualisations}

Les visualisations permettent de préciser la nature de la cavitation qui apparaît dans les entrefers des différentes pales. Nous reproduisons ici quelques photographies parmi les plus typiques, qui illustrent les caractéristiques les plus saillantes de la cavitation. La figure $l$ montre les visualisations de cavitation sur le rotor 2 pour deux géométries d'entrefer. On vérifie la forte cavitation qui apparait dans les entrefers à arêtes vives (pale 5). Le résultat le plus significatif est l'effet très bénéfique de l'arrondi de larête d'entrée sur la cavitation d'entrefer (pale 8).

L'effet du dévers sur la cavitation est également un des résultats les plus significatifs de cette étude. Les formes prises par la cavitation dans un entrefer de géométrie à arêtes vives sont montrées sur la figure la pour une pale ayant un dévers direct et sur la figure 2 pour un entrefer de mêmes caractéristiques monté sur une pale sans dévers (fig. 2a) ou avec dévers inverse (fig. 2b). On observe effectivement que la cavitation est bien moins intense pour le dévers inverse et bien plus intense pour le dévers direct par comparaison avec la configuration sans dévers. Ces tendances sont visibles aussi bien sur la cavitation de type vortex que sur celle de type entrefer.
Les visualisations procurent des informations complémentaires qu'il n'est pas possible de toutes illustrer ici par des photographies. Citons cependant que :

- (i) l'intensité de la cavitation de vortex décroît généralement lorsque la hauteur de jeu de l'entrefer augmente ; - (ii) aucune corrélation claire n'apparaît entre l'intensité de la cavitation d'entrefer et la hauteur du jeu; - (iii) des interactions entre cavitation de vortex et cavitation d'entrefer ont été notées.

\subsection{Mesures pariétales de pression}

Les mesures pariétales de pression, effectuées systématiquement, sont traitées numériquement. Les champs de pression, après moyennage des signaux sur 31 tours du rotor, sont tracés pour chaque pale sous la forme de cartes isovaleurs sur des domaines couvrant les entrefers et les espaces interaube. De telles cartes sont montrées sur la figure 3, pour les conditions d'essais de la figure $l$.

L'examen qualitatif des cartes isovaleurs de pression, comparées aux photographies, révèle la pertinence physique des signaux de pression. On superpose avec une excellente corrélation les cartes aux photographies. Cette corrélation, particulièrement bonne pour l'entrefer lui-même, se vérifie également pour l'écoulement dans l'espace interaube ; la signature du tourbillon marginal en terme de signal 


\section{R. LABORDE et al.}

de pression pariétale est tout à fait identifiable (la quantité reportée sur les cartes est l'écart de la pression mesurée à la pression amont). En comparant les cartes isovaleurs de pression avec les photographies, on constate, en accord avec l'expérience physique, que les minima de pression atteints pour une pale donnée sont d'autant plus bas que la cavitation est intense.

La comparaison quantitative des cartes isovaleurs de pression avec les seuils d'apparition de la cavitation d'entrefer est détaillée dans le rapport CERG 21.298.

\section{Conclusion}

Le caractère systématique de essais réalisés dans le cadre de cette étude a permis de cerner les effets produits par divers paramètres majeurs sur la cavitation d'entrefer et la cavitation de vortex, en géométrie confinée et en situation réelle de rotation. Le paramètre essentiel gouvernant la cavitation d'entrefer est la charge générée par le rotor.

Des effets tout à fait significatifs de réduction de la cavitation d'entrefer sont obtenus en modifiant la géométrie des entrefers et des pales. La cavitation d'entrefer est retardée de facon importante par un arrondi de l'arête sur l'intrados de l'entrefer. Cet effet est encore amplifié lorsque la pale présente un dévers en sens inverse. La modification de la cavitation d'entrefer avec la hauteur de jeu est, pour sa part, plus complexe. Les modifications de géométrie de l'entrefer envisagées dans cette étude ne réduisent pas la cavitation de vortex. En revanche, un dévers des pales en sens inverse agit favorablement sur la cavitation de vortex en géométrie confinée.

Sur le plan expérimental, les mesures nouvelles des champs de pression pariétale dans la zone balayée par la tête des pales, mises en œuvre dans cette étude, se révèlent être un outil précieux pour évaluer les phénomènes de cavitation d'entrefer et de cavitation de vortex. La cartographie des champs de pression est en excellent accord avec les observations. Les zones de cavitation sont retrouvées; les cartes de pression donnent un élément de comparaison fiable des différentes configurations; enfin, les tourbillons cavitants émis par le bout de pale possèdent une signature bien identifiable en paroi.

\section{Références}

Chantrel P. \& Lemonnier F. - " Hydroréacteurs pour tenue automatique d'immersion », Rapport Neyrtec 20.642, 1984, CD 885 .

FARRELl K.J. - "An investigation of end-wall vortex cavitation in a high Reynolds number axial flow pump ", Tech. Rep. TR 89-004, 1989, Pennsylvania State University.

GEARHART W.S. - "Tip clearance cavitation in shrouded underwater propulsors ", AIAA, J. of Aircraft, Vol. 3, $\mathrm{n}^{\circ} 2$, 1966, pp. 185-192.

LABORDE R. \& MORY M. - "Cavitation d'entrefer ", Rapports CERG R. 21.015 et R.21.030, 1988 (Contrat DRET 88/321).

LABORDE R. \& MORY M. - "Cavitation d'entrefer. Qualification du moyen d'essai. Rotor $n^{\circ} 1 »$, Rapport CERG R.21.200, 1990 (Contrat DRET 89/403).
Laborde R., Retailleau A. \& Mory M. - "Cavitation d'entrefer. Rotors $n^{\circ} 2$ et $n^{\circ} 3 "$, Rapport CERG R. 21.298, 1992 (Contrat DRET 90/518).

MÓller H. - "Cavitation dans l'entrefer des turbomachines rapides ", $Z$-VDI 79, n 39, 1935, pp. 1165-1169. (Traduction Neyrtec, 1978, R.20032).

Novoderezhrin R.A., Karelin V.Y. \& Demidov V.V.. " Experimental studies of tip clearance cavitation 》. Inst. of Civil Eng., Moscou, 1983.

PAPIR A.N. \& Golikov V.A. - "The evaluation of slot cavitation formation in hydraulic machines ", Hydroturbo Conf., Ostrava, Tchécoslovaquie, 1985, tome II, D2, pp. 127-134.

STRSCHELETZKY V.M. - « Les pertes d'entrefer dans les machines axiales, en particulier, dans les turbines Kaplan », Forschung auf dem gebiet des Ingenieurwesens, 21, $\mathrm{n}^{\circ} 4$, 1955, pp. 101-106 (trad. Neyrtec, 1978, R. 20.031). 